\title{
Virtual copies of semisimple Lie algebras in enveloping algebras of semidirect products and Casimir operators
}

\author{
R. Campoamor-Stursberg $\dagger$ \\ $\dagger$ Dpto. Geometría y Topología \\ Fac. CC. Matemáticas \\ Universidad Complutense de Madrid \\ Plaza de Ciencias, 3 \\ E-28040 Madrid, Spain \\ E-mail: rutwig@mat.ucm.es \\ S. G. Low \\ $\ddagger$ Austin, TX, USA \\ E-mail: Stephen.Low@alumni.utexas.net
}

\begin{abstract}
Given a semidirect product $\mathfrak{g}=\mathfrak{s} \uplus \mathfrak{r}$ of semisimple Lie algebras $\mathfrak{s}$ and solvable algebras $\mathfrak{r}$, we construct polynomial operators in the enveloping algebra $\mathcal{U}(\mathfrak{g})$ of $\mathfrak{g}$ that commute with $\mathfrak{r}$ and transform like the generators of $\mathfrak{s}$, up to a functional factor that turns out to be a Casimir operator of $\mathfrak{r}$. Such operators are said to generate a virtual copy of $\mathfrak{s}$ in $\mathcal{U}(\mathfrak{g})$, and allow to compute the Casimir operators of $\mathfrak{g}$ in closed form, using the classical formulae for the invariants of $\mathfrak{s}$. The behavior of virtual copies with respect to contractions of Lie algebras is analyzed. Applications to the class of Hamilton algebras and their inhomogeneous extensions are given.
\end{abstract}

PACS numbers: 02.20Sv, 02.20Qs 


\section{Introduction}

Casimir operators of Lie algebras and their generalizations constitute an important tool in dealing with various branches of theoretical physics. Typically their appear in connection with quantum numbers depicting the main properties of a system. Since the eigenvalues of such operators characterize irreducible representations and constitute measurable physical quantities, they provide natural labels for classification schemes and characterization of states. Further, connections between symmetries and dynamical properties of a system lead to the possibility of expressing Hamiltonians and deducing mass formulae from the Casimir operators of the involved Lie algebras. This fact has been extensively used in the algebraic models in atomic and nuclear physics [1]. Applications of invariants of Lie algebras to phenomenological aspects of a theory have also been shown to be useful, as given for example by the cubic $S U_{c}(3)$-operator in the confinement problem of the non-relativistic quark model or the construction of the relevant operators in $N=2$ supersymmetric models by means of Casimir operators [2].

Classical Casimir operators are operators from the universal enveloping algebra $\mathcal{U}(\mathfrak{g})$ that commute with all elements of the Lie algebra, and therefore lead to the concept of symmetry. For semisimple Lie algebras $\mathfrak{s}$, the problem of finding the centre of $\mathcal{U}(\mathfrak{s})$ has been solved long ago [3, 4, 5], using the important structural properties that are essential for their classification [6]. Although polynomial invariants of an arbitrary Lie algebra always belong to the centre of $\mathcal{U}(\mathfrak{g})$, for non-semisimple algebras this direct approach is quite complicated in practice, due to the absence of structural properties like the Killing form and the difficulties of their representation theory. For these types of algebras, an analytic approach has shown to be more effective [7, 8, 9, 10, 11, 12, 13].

However, the construction of Casimir operators by means of enveloping algebras can be adapted well to semidirect products $\mathfrak{w} \mathfrak{s}=\mathfrak{s} \vec{\oplus}_{R} \mathfrak{w}(n)$ of simple Lie algebras $\mathfrak{s}$ with the Heisenberg-Weyl algebra $\mathfrak{w}(n)$. The main idea is to find quadratic operators in the enveloping algebra that transform like the generators of the Levi subalgebra $\mathfrak{s}$ and commute with the generators of $\mathfrak{w}(n)$. In this manner, the Casimir operators can be easily recovered using the classical formulae for the invariants of $\mathfrak{s}$. This compatibility is partially explained by the simplicity of the boson realizations for these algebras, and generally fails for radicals other than the Heisenberg algebras.

In this work, we propose a natural generalization of this method to determine the Casimir operators of wide classes of semidirect products of semisimple and solvable Lie algebras. The main difference with the $\mathfrak{w} \mathfrak{s}$ case is that the operators in the enveloping algebra are of order higher than quadratic. These operators commute with the generator of the radical $\mathfrak{r}$, and transform like the generators of the Levi subalgebra $\mathfrak{s}$, up to a functional factor given by a Casimir operator of $\mathfrak{g}$ depending only on the generators of the radical. It follows that the normalized operators do not live properly in the enveloping algebra of $\mathfrak{g}$, but in its fraction field [8]. For this reason such copies will be called virtual. This modification does however not affect the computation of the Casimir 
operators using the formulae for the invariants of $\mathfrak{s}$.

As an application of this method, we obtain the Casimir operators for the inhomogeneous Hamilton algebras $\mathrm{IHa}(\mathrm{N})$ and all its central extensions in arbitrary dimension. These algebras have been used in the group theoretical analysis of noninertial states in Quantum Mechanics [14]. Some unanswered questions formulated in [15] concerning the number and form of their invariants are solved simultaneously for all values of $N$.

Finally, given a contraction $\mathfrak{g} \rightsquigarrow \mathfrak{g}^{\prime}$, we analyze under which conditions the operators spanning a virtual copy of the Levi subalgebra $\mathfrak{s} \subset \mathfrak{g}$ in $\mathcal{U}(\mathfrak{g})$ can be contracted to operators generating a copy of the same semisimple algebra in the enveloping algebra of the contraction $\mathfrak{g}^{\prime}$.

\section{Invariants of Lie algebras. Maurer-Cartan equations}

Given a Lie algebra $\mathfrak{g}=\left\{X_{1}, . ., X_{n} \mid\left[X_{i}, X_{j}\right]=C_{i j}^{k} X_{k}\right\}$ in terms of generators and commutation relations, we are primarily interested in (polynomial) operators $C_{p}=$ $\alpha^{i_{1} . . i_{p}} X_{i_{1}} . . X_{i_{p}}$ in the generators of $\mathfrak{s}$ such that the constraint $\left[X_{i}, C_{p}\right]=0,(i=1, . ., n)$ is satisfied. Such an operator can be shown to lie in the centre of the enveloping algebra of $\mathfrak{g}$, and is traditionally referred to as Casimir operator. For semisimple Lie algebras, the determination of Casimir operators can be done using structural properties [4]. However, for non-semisimple Lie algebras the relevant invariant functions are often rational or even transcendental functions [12]. This suggest to develop a method in order to cover arbitrary Lie algebras. One convenient approach is the analytical realization. The generators of the Lie algebra $\mathfrak{s}$ are realized in the space $C^{\infty}\left(\mathfrak{g}^{*}\right)$ by means of the differential operators:

$$
\widehat{X}_{i}=C_{i j}^{k} x_{k} \frac{\partial}{\partial x_{j}}
$$

where $\left\{x_{1}, . ., x_{n}\right\}$ is a dual basis of $\left\{X_{1}, . ., X_{n}\right\}$. The invariants of $\mathfrak{g}$ (in particular, the Casimir operators) are solutions of the following system of partial differential equations:

$$
\widehat{X}_{i} F=0, \quad 1 \leq i \leq n .
$$

Whenever we have a polynomial solution of (2), the symmetrization map defined by

$$
\operatorname{Sym}\left(x_{i_{1}}^{a_{1}} . . x_{i_{p}}^{a_{p}}\right)=\frac{1}{p !} \sum_{\sigma \in S_{p}} x_{\sigma\left(i_{1}\right)}^{a_{1}} . . x_{\sigma\left(i_{p}\right)}^{a_{p}}
$$

allows to recover the Casimir operators in their usual form, i.e, as elements in the centre of the enveloping algebra of $\mathfrak{g}$, after replacing the variables $x_{i}$ by the corresponding generator $X_{i}$ [8]. A maximal set of functionally independent invariants is usually called a fundamental basis. The number $\mathcal{N}(\mathfrak{g})$ of functionally independent solutions of (2) is obtained from the classical criteria for differential equations, and is given by:

$$
\mathcal{N}(\mathfrak{g}):=\operatorname{dim} \mathfrak{g}-\sup _{x_{1}, . ., x_{n}} \operatorname{rank}\left(C_{i j}^{k} x_{k}\right),
$$


where $A(\mathfrak{g}):=\left(C_{i j}^{k} x_{k}\right)$ is the matrix associated to the commutator table of $\mathfrak{g}$ over the given basis.

The use of differential forms has turned out to be useful not only to reformulate formula (4), but also to compute Casimir operators in some situations [16]. In terms of the Maurer-Cartan equations, the Lie algebra $\mathfrak{g}$ is described as follows: Given the structure tensor $\left\{C_{i j}^{k}\right\}$ over the basis $\left\{X_{1}, . ., X_{n}\right\}$, the identification of the dual space $\mathfrak{g}^{*}$ with the left-invariant 1-forms on the simply connected Lie group whose algebra is isomorphic to $\mathfrak{g}$ allows to define an exterior differential $d$ on $\mathfrak{g}^{*}$ by

$$
d \omega\left(X_{i}, X_{j}\right)=-C_{i j}^{k} \omega\left(X_{k}\right), \omega \in \mathfrak{g}^{*} .
$$

This coboundary operator $d$ allows us to rewrite $\mathfrak{g}$ as a closed system of 2 -forms

$$
d \omega_{k}=-C_{i j}^{k} \omega_{i} \wedge \omega_{j}, 1 \leq i<j \leq \operatorname{dim}(\mathfrak{g}),
$$

called the Maurer-Cartan equations of $\mathfrak{g}$. The closeness condition $d^{2} \omega_{i}=0$ for all $i$ is equivalent to the Jacobi condition. To reformulate equation (44) in terms of differential forms, we consider the linear subspace $\mathcal{L}(\mathfrak{g})=\mathbb{R}\left\{d \omega_{i}\right\}_{1 \leq i \leq \operatorname{dim} \mathfrak{g}}$ of $\bigwedge^{2} \mathfrak{g}^{*}$ generated by the 2 -forms $d \omega_{i}$ [17]. In particular, $\operatorname{dim} \mathcal{L}(\mathfrak{g})=\operatorname{dim}(\mathfrak{g})$ is equivalent to the condition $\operatorname{dim}(\mathfrak{g})=\operatorname{dim}[\mathfrak{g}, \mathfrak{g}]$. Considering a generic element $\omega=a^{i} d \omega_{i} \quad\left(a^{i} \in \mathbb{R}\right)$ of $\mathcal{L}(\mathfrak{g})$, then we can always find a positive integer $j_{0}(\omega) \in \mathbb{N}$ such that $\bigwedge^{j_{0}(\omega)} \omega \neq 0$ and $\bigwedge^{j_{0}(\omega)+1} \omega \equiv 0$. Thus defining the quantity $j_{0}(\mathfrak{g})$ as the maximal rank of generic elements,

$$
j_{0}(\mathfrak{g})=\max \left\{j_{0}(\omega) \mid \omega \in \mathcal{L}(\mathfrak{g})\right\},
$$

we get a numerical invariant of the Lie algebra $\mathfrak{g}$ that can be used to rewrite equation (4) as

$$
\mathcal{N}(\mathfrak{g})=\operatorname{dim} \mathfrak{g}-2 j_{0}(\mathfrak{g}) .
$$

The scalar $j_{0}(\mathfrak{g})$ further determines the number of internal labels necessary to describe a general irreducible representation of $\mathfrak{g}[3$, ,, 17$]$.

\section{Virtual copies of semisimple Lie algebras}

Ch. Quesne developed in [18] a method based on enveloping algebras to compute the Casimir operators of semidirect products $\mathfrak{s} \vec{\oplus}_{R} \mathfrak{w}(n)$ of simple Lie algebras $\mathfrak{s}$ with Heisenberg-Weyl algebras $\mathfrak{w}(n)$ spanned by $n$ pairs of boson operators $b_{i}, b_{j}^{\dagger}$ and the unit operator $\mathbb{I}$. Since the Casimir operators of simple Lie algebras are known [4], the method reduces essentially to find polynomials in the generators of $\mathfrak{s} \vec{\oplus}_{R} \mathfrak{w}(n)$ that span a copy of $\mathfrak{s}$ in the enveloping algebra of $\mathfrak{s} \vec{\oplus}_{R} \mathfrak{w}(n)$. More specifically, taking the basis $\left\{E_{i j}, b_{i}, b_{j}^{\dagger}, \mathbb{I}\right\}$ of $\mathfrak{s} \vec{\oplus}_{R} \mathfrak{w}(n)$, where the $E_{i j}$ spans the Levi part $\mathfrak{s}$, the author constructed quadratic operators

$$
E_{i j}^{\prime}=E_{i j} \mathbb{I}-b_{i}^{\dagger} b_{j}
$$

satisfying the constraints

$$
\left[E_{i j}^{\prime}, b_{k}^{\dagger}\right]=\left[E_{i j}^{\prime}, b_{k}\right]=0,\left[E_{i j}, E_{k l}^{\prime}\right]=\left[E_{i j}, E_{k l}\right]^{\prime} .
$$


These identities ensure that the $E_{i j}^{\prime}$ transform in a similar way to the original generators of $\mathfrak{s}$, i.e., satisfy the commutation relation

$$
\left[E_{i j}^{\prime}, E_{k l}^{\prime}\right]=\mathbb{I}\left[E_{i j}, E_{k l}\right]^{\prime},
$$

and therefore span a copy of the latter in the enveloping algebra of $\mathfrak{s} \vec{\oplus}_{R} \mathfrak{w}(n) \ddagger$ Now, using the expression for a Casimir operator of order $p$ of $\mathfrak{s}$,

$$
C_{p}=\sum_{i_{1} . . i_{p}} E_{i_{1} i_{2}} \ldots E_{i_{p} i_{1}}
$$

the invariant for the corresponding copy $\mathfrak{s}^{\prime}$ in the enveloping algebra $\mathcal{U}\left(\mathfrak{s} \vec{\oplus}_{R} \mathfrak{w}(n)\right)$ is simply

$$
C_{p}^{\prime}=\sum_{i_{1} . . i_{p}} E_{i_{1} i_{2}}^{\prime} \ldots E_{i_{p} i_{1}}^{\prime}
$$

As a consequence of equation (10), this operator satisfies the identities

$$
\left[C_{p}^{\prime}, b_{k}^{\dagger}\right]=\left[C_{p}^{\prime}, b_{k}\right]=\left[C_{p}^{\prime}, E_{i j}\right]=0,
$$

proving that $C_{p}^{\prime}$ is a Casimir operator of the Lie algebra $\mathfrak{s} \vec{\oplus}_{R} \mathfrak{w}(n)$. Independence of the invariants computed by this method follows easily by direct verification.

In this case, the validity of the method relies heavily on the specific structure of these semidirect products $\$$ One can however ask whether a similar ansatz can be made for other types of semidirect products, including Lie algebras whose radical is not necessarily a nilpotent algebra. It seems reasonable to require that such a Lie algebra $\mathfrak{g}$ satisfies the inequality $\mathcal{N}(\mathfrak{g}) \geq \mathcal{N}(\mathfrak{s})$, where $\mathfrak{s}$ is the Levi subalgebra of $\mathfrak{g}$, although it is not ensured a priori that the generalization of (9) leads to functionally independent invariants of $\mathfrak{g}$.

The general idea to expand the ansatz of [18] to more wide types of semidirect products is to replace the operators (9) by more general functions of the generators, and then analyze under which constraints these operators transform in similar way to the identities (10). To this extent, we start from an unspecified non-semisimple Lie algebra $\mathfrak{g}$ with Levi decomposition $\mathfrak{g}=\mathfrak{s} \vec{\oplus}_{R} \mathfrak{r}$, where $\mathfrak{s}$ denotes the Levi subalgebra and $\mathfrak{r}$ the radical $\llbracket$ Let $\left\{X_{1}, . ., X_{n}, Y_{1}, . ., Y_{m}\right\}$ be a basis such that $\left\{X_{1}, . ., X_{n}\right\}$ spans $\mathfrak{s}$ and $\left\{Y_{1}, . ., Y_{m}\right\}$ spans $\mathfrak{r}$. We further suppose that the structure tensor in $\mathfrak{s}$ is given by

$$
\left[X_{i}, X_{j}\right]=C_{i j}^{k} X_{k}
$$

We now define operators $X_{i}^{\prime}$ in the enveloping algebra of $\mathfrak{g}$ by means of

$$
X_{i}^{\prime}=X_{i} f\left(Y_{1}, . ., Y_{m}\right)+P_{i}\left(Y_{1}, . ., Y_{m}\right),
$$

$\ddagger$ Observe that, since $\mathbb{I}$ is the identity operator, it can be skipped from equation (11).

$\S$ Specifically, it uses that the semidirect product $\mathfrak{s} \vec{\oplus}_{R} \mathfrak{w}(n)$ has a one dimensional centre generated by the identity operator $\mathbb{I}$.

$\|$ Recall that the radical is the maximal solvable ideal of $\mathfrak{g}$, while the Levi subalgebra is the maximal semisimple subalgebra. 
where $P_{i}$ is a homogeneous polynomial of degree $k$ and $f$ is homogeneous of degree $k-1$. In order to generalize the method of [18], we require the constraints

$$
\begin{aligned}
{\left[X_{i}^{\prime}, Y_{k}\right] } & =0, \\
{\left[X_{i}^{\prime}, X_{j}\right] } & =\left[X_{i}, X_{j}\right]^{\prime}:=C_{i j}^{k}\left(X_{k} f+P_{k}\right) .
\end{aligned}
$$

to be satisfied for all generators. We thus have to analyze the conditions that the functions $f$ and $P_{i}$ must satisfy in order for these equations to hold. After development, equation (17) can be rewritten as

$$
\left[X_{i}^{\prime}, Y_{j}\right]=\left[X_{i} f, Y_{j}\right]+\left[P_{i}, Y_{j}\right]=X_{i}\left[f, Y_{j}\right]+\left[X_{i}, Y_{j}\right] f+\left[P_{i}, Y_{j}\right] .
$$

Since the quantities involved are homogeneous in the variables of $\mathfrak{s}$ and $\mathfrak{r}$, we can reorder the terms according to their degree. Observe that $X_{i}\left[f, Y_{j}\right]$ is a homogeneous polynomial of degree $k-1$ in the variables $\left\{Y_{1}, . ., Y_{m}\right\}$, while $\left[X_{i}, Y_{j}\right] f+\left[P_{i}, Y_{j}\right]$ is of degree $k$. Therefore the terms must fulfill the following identities

$$
\begin{aligned}
& {\left[f, Y_{j}\right]=0,} \\
& {\left[X_{i}, Y_{j}\right] f+\left[P_{i}, Y_{j}\right]=0 .}
\end{aligned}
$$

From the first one we conclude that $f$ must be a Casimir operator of the radical $\mathfrak{r}$. The second condition shows the obstruction to the $P_{i}^{\prime} s$ to be also invariants of $\mathfrak{r}$. Observe in particular that if $Y_{j}$ commutes with the generator $X_{i}$ of $\mathfrak{s}$, the it must commute with the operator $X_{i}^{\prime}$. We now focus on equation (18). Development of the latter leads to the identity

$$
\left[X_{i}^{\prime}, X_{j}\right]=\left[X_{i}, X_{j}\right] f-X_{i}\left[X_{j}, f\right]+\left[P_{i}, X_{j}\right]
$$

The polynomial $\left[X_{i}, X_{j}\right] f-X_{i}\left[X_{j}, f\right]$ is homogeneous of degree $k-1$ in the variables of $\mathfrak{r}$ and degree one in the variables of $\mathfrak{s}$, while $\left[P_{i}, X_{j}\right]$ is of degree zero in the latter set of variables. This implies that the system

$$
\begin{aligned}
& {\left[X_{i}, X_{j}\right] f-X_{i}\left[X_{j}, f\right]=C_{i j}^{k} X_{k} f} \\
& {\left[P_{i}, X_{j}\right]=C_{i j}^{k} P_{k}}
\end{aligned}
$$

must be satisfied for arbitrary indices $i, j$. Because of (15), the first equation reduces to

$$
X_{i}\left[X_{j}, f\right]=0,
$$

and since this holds for all generators of $\mathfrak{s}$, we deduce that $f$ must be a Casimir operator of $\mathfrak{g}$ that depends only on the variables of the radical $\mathfrak{r}$. The second condition tells that the $P_{i}^{\prime} s$ transform under the $X_{j}^{\prime} s$ like the generators of the semisimple part $\mathfrak{s}$.

Taken together, it follows from these relations that the operators $X_{i}^{\prime}$ transform like the generators of $\mathfrak{s}$, up to the additional factor $f$ given by a Casimir operator. More precisely, for any indice $i, j$ we have the commutator

$$
\begin{aligned}
{\left[X_{i}^{\prime}, X_{j}^{\prime}\right] } & =\left[X_{i} f+P_{i}, X_{j} f+P_{j}\right]=\left[X_{i} f+P_{i}, X_{j} f\right]+\left[X_{i} f+P_{i}, P_{j}\right] \\
& =C_{i j}^{k} X_{k} f^{2}+C_{i j}^{k} P_{k} f+\left[X_{i}^{\prime}, P_{j}\right]=f\left[X_{i}, X_{j}\right]^{\prime},
\end{aligned}
$$

where the last equality is a consequence of (17). 
Because of this factor $f$, we cannot in general find operators $X_{i}^{\prime \prime}$ in the enveloping algebra of $\mathfrak{g}$ satisfying (17) and (18) and such that

$$
\left[X_{i}^{\prime \prime}, X_{j}^{\prime \prime}\right]=\left[X_{i}, X_{j}\right]^{\prime \prime}
$$

For this reason we will say that the operators $X_{i}^{\prime}$ generate a virtual copy of $\mathfrak{s}$ in the enveloping algebra of $\mathfrak{g}$. We remark that only for the case where $f$ can be identified with an identity operator, as happens for Heisenberg radical, this virtual copy actually generates a copy in $\mathcal{U}(\mathfrak{g})$. However, this functional factor $f$ does not affect the validity of the method to compute Casimir operators of $\mathfrak{g}$ from those of the Levi part $\mathfrak{s}$.

Theorem 1 Let $\mathfrak{s}$ be the Levi part of a Lie algebra $\mathfrak{g}$ and let $X_{i}^{\prime}=X_{i} f\left(Y_{1}, . ., Y_{m}\right)+$ $P_{i}\left(Y_{1}, . ., Y_{m}\right)$ be polynomials in the generators of $\mathfrak{g}$ satisfying equations (20) and (22). If $C=\sum \alpha^{i_{1} . i_{p}} X_{i_{1}} . . X_{i_{p}}$ is a Casimir operator of degree $p$ of $\mathfrak{s}$, then $C^{\prime}=\sum \alpha^{i_{1} . . i_{p}} X_{i_{1}}^{\prime} . . X_{i_{p}}^{\prime}$ is a Casimir operator of $\mathfrak{g}$ of degree $p \operatorname{deg} f$. Moreover, $\mathcal{N}(\mathfrak{g}) \geq \mathcal{N}(\mathfrak{s})+1$.

Proof. It is clear from equations (17) and (18) that if $C=\sum \alpha^{i_{1} . . i_{p}} X_{i_{1}} . . X_{i_{p}}$ is a Casimir operator of $\mathfrak{s}$, then $C^{\prime}=\sum \alpha^{i_{1} . . i_{p}} X_{i_{1}}^{\prime} . . X_{i_{p}}^{\prime}$ is a Casimir operator of $\mathfrak{g}$. To prove the second assertion, we observe that, since $X_{i}^{\prime}=X_{i} f+P_{i}$, we can rewrite $C^{\prime}$ as

$$
C^{\prime}=\sum \alpha^{i_{1} . . i_{p}}\left(X_{i_{1}} . . X_{i_{p}} f^{p}+\sum_{t=1}^{p} \sum_{j_{1}, . ., j_{t}=i_{1}}^{i_{p}} X_{i_{1}} . . P_{j_{1}} . . P_{j_{t}} . . X_{i_{p}} f^{p-t}\right) .
$$

For any $1 \leq t \leq p$, the polynomial

$$
F_{\left[j_{1}, . . j_{t}\right]}=\sum_{j_{1}, . ., j_{t}=i_{1}}^{i_{p}} X_{i_{1}} . . P_{j_{1}} . . P_{j_{t}} . . X_{i_{p}} f^{p-t}
$$

is homogeneous of degree $p-t$ in the generators $\left\{X_{1}, . ., X_{n}\right\}$. In particular, $C_{p}^{\prime}$ contains $C_{p}$ as the term of maximal degree in the latter set of generators. Let $\left\{C_{1}, . ., C_{l}\right\}$ be a set of independent Casimir operators of $\mathfrak{s}$. We can thus find indices $k_{1}, . ., k_{l}$ such that the associated Jacobian does not vanish:

$$
\frac{\partial\left\{C_{1}, . ., C_{l}\right\}}{\partial\left\{X_{k_{1}}, . ., X_{k_{l}}\right\}} \neq 0
$$

Let $d_{i}=\operatorname{deg} C_{i}$ denote the degree of each invariant. We now rewrite $\left\{C_{1}^{\prime}, . ., C_{l}^{\prime}\right\}$ according to (25), and compute the Jacobian with respect to the generators $\left\{X_{k_{1}}, . ., X_{k_{l}}\right\}$. We explicitly obtain

$\frac{\partial\left\{C_{1}^{\prime}, . ., C_{l}^{\prime}\right\}}{\partial\left\{X_{k_{1}}, . ., X_{k_{l}}\right\}}=\operatorname{det}\left(\begin{array}{ccc}\frac{\partial C_{1}}{X_{k_{1}}} f^{d_{1}}+\sum_{t=1}^{d_{1}} \frac{\partial}{\partial X_{k_{1}}} F_{1,\left[j_{1}, ., j_{t}\right]} & . . & \frac{\partial C_{1}}{X_{k_{l}}} f^{d_{1}}+\sum_{t=1}^{d_{1}} \frac{\partial}{\partial X_{k_{l}}} F_{1,\left[j_{1}, . . j_{t}\right]} \\ \vdots & & \vdots \\ \frac{\partial C_{l}}{X_{k_{1}}} f^{d_{l}}+\sum_{t=1}^{d_{l}} \frac{\partial}{\partial X_{k_{1}}} F_{l,\left[j_{1}, ., j_{t}\right]} & . . & \frac{\partial C_{l}}{X_{k_{l}}} f^{d_{l}}+\sum_{t=1}^{d_{l}} \frac{\partial}{\partial X_{k_{l}}} F_{l,\left[j_{1}, ., j_{t}\right]}\end{array}\right)$.

Using the properties of determinants, it can be easily seen that (27) simplifies to

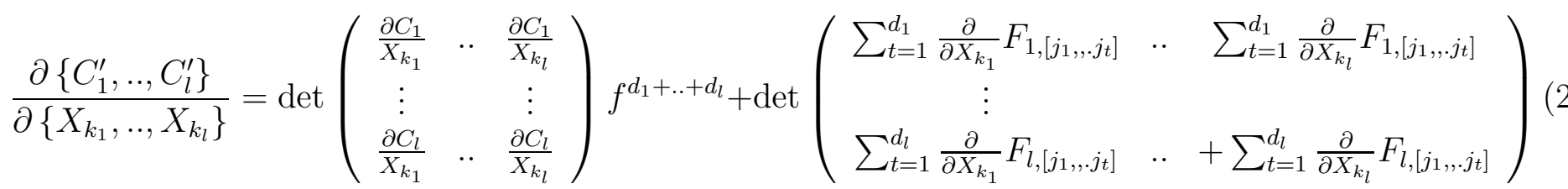

$$
(28
$$

I The operators $X_{i}^{\prime \prime}=X_{i}+\frac{1}{f} P_{i}$ would belong to the fraction field of the enveloping algebra $\mathcal{U}(\mathfrak{g})[8]$. 
Since the first term of the latter equation coincides with $\frac{\partial\left\{C_{1}, . ., C_{l}\right\}}{\partial\left\{X_{k_{1}}, \ldots, X_{k_{l}}\right\}} f^{d_{1}+. .+d_{l}}$ and has maximal degree in the $X_{i}^{\prime}$ s, condition (26) ensures that (27) does not vanish, i.e., the operators $\left\{C_{1}^{\prime}, . ., C_{l}^{\prime}\right\}$ are independent. Finally, $f$ being a Casimir operator of $\mathfrak{g}$ depending only on the variables of the radical, we get $\mathcal{N}(\mathfrak{s})+1 \leq \mathcal{N}(\mathfrak{g})$.

Hence the independence of the invariants constructed starting from those of the Levi part $\mathfrak{s}$ is automatically ensured by the conditions (17) and (18). This result further allows us to obtain a criterion concerning the existence of virtual copies of semisimple algebras in enveloping algebras of semidirect products. The proof is a direct consequence of the previous argument.

Proposition 1 Let $\mathfrak{s}$ be the Levi subalgebra of the Lie algebra $\mathfrak{g}$. If $\mathcal{N}(\mathfrak{g}) \leq \mathcal{N}(\mathfrak{s})$, then no virtual copy of $\mathfrak{s}$ in the enveloping algebra of $\mathfrak{g}$ exists.

This means specifically that for semidirect products with less invariants than those of its Levi part, the latter provides no valid information to simplify the computation of the Casimir invariants. In these cases, an approach by enveloping algebras is specially difficult, since the operators have to be found directly. The ansatz further allows to establish some restrictions on the structure of the radical $\mathfrak{r}$ of such semidirect products.

Proposition 2 Let $\mathfrak{g}=\mathfrak{s} \vec{\oplus}_{R} \mathfrak{r}$ admit a (virtual) copy of $\mathfrak{s}$ in the enveloping algebra $\mathcal{U}(\mathfrak{g})$ generated by operators of type (16)). Then the radical $\mathfrak{r}$ is not Abelian.

The proof is a direct consequence of equation (20). In fact, if the operators $X_{i j}^{\prime}=X_{i}^{\prime} f\left(Y_{1}, . ., Y_{m}\right)+P_{i}\left(Y_{1}, . ., Y_{m}\right)$ satisfy the constraint (17), by the second equation of (201) we have that

$$
\left[X_{i}, Y_{j}\right] f+\left[P_{i}, Y_{j}\right]=0 .
$$

If $\mathfrak{r}$ were Abelian, then obviously $\left[P_{i}, Y_{j}\right]=0$ for all indices $i, j$, and this would imply, since $f \neq 0$, that $\left[X_{i}, Y_{j}\right]=0$ for all generators $X_{i}, Y_{j}$. But this tells that the representation $R$ of $\mathfrak{s}$ on $\mathfrak{r}$ is trivial, hence the algebra $\mathfrak{g}$ reduces to a direct sum $\mathfrak{s} \oplus \mathfrak{r}$.

\section{Invariants of the inhomogeneous Hamilton algebra}

The Hamilton algebra and its various extensions arise naturally in the analysis of relativity groups for noninertial states in the quantum mechanical frame [15]. It is known that projective representations on physical states lead to the conclusion that relativity groups arise as subgroups of the automorphism group of the Heisenberg algebra $\mathfrak{h}_{N}$. In particular, it follows that the Hamilton group, which is isomorphic to the semidirect product of $\mathfrak{s o}(N)$ with the Heisenberg algebra $\mathfrak{h}_{N}$, coincides with the relativity group for noninertial frames in classical Hamilton mechanics [19]. Its Lie algebra $\mathrm{Ha}(N)$ is given, over the basis $\left\{J_{i j}, G_{k}, F_{k}, R\right\}$, by the brackets

$$
\begin{aligned}
& {\left[J_{i j}, J_{k l}\right]=\delta_{i}^{l} J_{j k}+\delta_{j}^{k} J_{i l}-\delta_{j}^{l} J_{i k}-\delta_{i}^{k} J_{j l}, \quad\left[G_{i}, F_{j}\right]=\delta_{i}^{j} R .} \\
& {\left[J_{i j}, G_{k}\right]=-\delta_{i}^{k} G_{j}+\delta_{k}^{j} G_{i}, \quad\left[J_{i j}, F_{k}\right]=-\delta_{i}^{k} F_{j}+\delta_{k}^{j} F_{i},}
\end{aligned}
$$


The action of $\mathfrak{s o}(N)$ over the Heisenberg radical is easily seen to be described by the fundamental $N$-dimensional tensor representation $\Lambda$ of $\mathfrak{s o}(N)$. Therefore its dimension is $\operatorname{dim} H a(N)=\frac{1}{2} N(N+3)+1$. Following [18], this algebra has $\left[\frac{N}{2}\right]+1$ Casimir operators. In this case, the operators

$$
J_{i j}^{\prime}=J_{i j} R+G_{i} F_{j}-G_{j} F_{i}
$$

generate the searched copy of $\mathfrak{s o}(N)$. Thus the Casimir operators of $\mathrm{Ha}(\mathrm{N})$ are obtained as the symmetrized polynomials of the functions $C_{k}$ arising as coefficients of the polynomial

$$
\operatorname{Cas}_{N}=\operatorname{det}\left|A_{N}-T \operatorname{Id}_{N}\right|=\sum_{l=1}^{N} C_{2 l} T^{2 N-2 l}+T^{2 N},
$$

where $A_{N}$ is the $N \times N$ matrix defined by

$$
A_{N}=\left(\begin{array}{cccc}
0 & J_{12}^{\prime} & \ldots & J_{1 N}^{\prime} \\
-J_{12}^{\prime} & 0 & \ldots & J_{2 N}^{\prime} \\
\vdots & \vdots & & \vdots \\
-J_{1 N}^{\prime} & -J_{2 N}^{\prime} & \ldots & 0
\end{array}\right) .
$$

An immediate question that arises naturally from this is whether the various extensions of the Hamilton algebra [15, 19] can be analyzed by means of virtual copies of $\mathfrak{s o}(N)$ in the corresponding enveloping algebras. In particular, insertion of the operators generating the corresponding copies into matrix (30) would provide the fundamental set of invariants for each of these extensions for arbitrary dimension $N$.

We first consider the inhomogeneous Hamilton algebra $\mathrm{IHa}(\mathrm{N})$, which is the first step in the study of quantum noninertial states [14]. This (non-central) extension is obtained by addition of the generators $\left\{Q_{k}, P_{k}, E, T\right\}$ to those of $H a(N)$ : The brackets are those of (29), to which the following are added:

$$
\begin{aligned}
& {\left[J_{i j}, Q_{k}\right]=-\delta_{i}^{k} Q_{j}+\delta_{k}^{j} Q_{i}, \quad\left[J_{i j}, P_{k}\right]=-\delta_{i}^{k} P_{j}+\delta_{k}^{j} P_{i}, \quad\left[G_{i}, Q_{j}\right]=\delta_{i}^{j} T,} \\
& {\left[F_{i}, P_{j}\right]=\delta_{i}^{j} T, \quad\left[E, G_{i}\right]=-P_{i}, \quad\left[E, F_{i}\right]=Q_{i}, \quad[E, R]=2 T .}
\end{aligned}
$$

It is observed that $R$ is no more a central element, but now $T$ plays the role of central charge. In particular, the radical is a solvable non-nilpotent Lie algebra. The first question that arises is how many invariants this algebra $\mathrm{IHa}(\mathrm{N})$ has.

Lemma 1 For any $N \geq 3$ following identity holds:

$$
\mathcal{N}(\text { IHa }(N))=\left[\frac{N}{2}\right]+1 .
$$

Proof. We consider the Maurer-Cartan equations (MC) of the algebra to prove it [17. Let $\left\{\omega_{i j}, \theta_{k}, \eta_{k}, \varphi_{k}, \chi_{k}, \omega_{R}, \omega_{E}, \omega_{T}\right\}$ be the dual basis to $\left\{J_{i j}, G_{k}, F_{k}, Q_{k}, P_{k}, R, E, T\right\}$. The Maurer-Cartan equations are easily seen to be

$$
\begin{aligned}
& d \omega_{i j}=-\sum_{k=1}^{N} \omega_{i k} \wedge \omega_{j k}, d \theta_{k}=\sum_{j=1}^{N} \omega_{k j} \wedge \theta_{j}, d \eta_{k}=\sum_{j=1}^{N} \omega_{k j} \wedge \eta_{j}, \\
& d \varphi_{k}=\sum_{j=1}^{N} \omega_{k j} \wedge \varphi_{j}+\omega_{E} \wedge \eta_{k}, d \chi_{k}=\sum_{j=1}^{N} \omega_{k j} \wedge \chi_{j}-\omega_{E} \wedge \theta_{k}, \\
& d \omega_{R}=\sum_{k=1}^{N} \theta_{k} \wedge \eta_{k}, d \omega_{T}=\sum_{k=1}^{N}\left(\theta_{k} \wedge \varphi_{k}+\eta_{k} \wedge \chi_{k}\right)+2 \omega_{E} \wedge \omega_{R}, \\
& d \omega_{E}=0
\end{aligned}
$$


Any generic element $\omega \in \mathcal{L}(\mathfrak{g})$ :

$$
\omega=a^{i j} d \omega_{i j}+b_{1}^{k} d \theta_{k}+b_{2}^{k} d \eta_{k}+b_{3}^{k} d \varphi_{k}+b_{4}^{k} d \chi_{k}+b_{5} d \omega_{R}+b_{6} d \omega_{T},
$$

where $a^{i j}, b_{j}^{k} \in \mathbb{R}$ are arbitrary constants, can be decomposed as $\omega=\xi_{1}+\xi_{2}$, where $\xi_{1}=a^{i j} d \omega_{i j} \in \mathcal{L}(\mathfrak{s})$ and $\xi_{2}=b_{1}^{k} d \theta_{k}+b_{2}^{k} d \eta_{k}+b_{3}^{k} d \varphi_{k}+b_{4}^{k} d \chi_{k}+b_{5} d \omega_{R}+b_{6} d \omega_{T} \in \mathcal{L}(\mathfrak{r})$. A routine but tedious computation shows that

$$
\bigwedge^{p}\left(\xi_{2}-b_{6} d \omega_{T}\right)=0, p \geq 2 N .
$$

This equation follows essentially from the fact that $\bigwedge^{N} d \theta_{k}=\bigwedge^{N} d \eta_{k}=\bigwedge^{N+1} d \varphi_{k}=$ $\Lambda^{N+1} d \chi_{k}=0$ and that the terms in the MC equations of these generators involve all rotation 1-forms $\omega_{i j}$. We further observe that $d \omega_{T}$ involves all 1-forms associated to generators of the radical with the exception of $T$, and satisfies the following identity $2 N+1$

$$
\bigwedge d \omega_{T}= \pm 2(2 N+1) ! \theta_{1} \wedge . . \wedge \theta_{N} \wedge \varphi_{1} \wedge . . \wedge \varphi_{N} \wedge \chi_{1} \wedge . . \wedge \chi_{N} \wedge \eta_{1} \wedge . . \wedge \eta_{N} \wedge \omega_{R} \wedge \omega_{E} .
$$

This proves that the equality $j_{0}\left(\xi_{2}\right)=2 N+1$ is satisfied only if $b_{6} \neq 0$. For this reason we fix $\xi_{2}=d \omega_{T}$. This choice has the advantage that no element of the dual space to $\mathfrak{s o}(N)$ appears in the expression of $\xi_{2}$. We now consider the Maurer-Cartan equations of the Levi part. Since $j_{0}(\mathfrak{s o}(N))=\frac{1}{2}\left(\frac{N(N-1)}{2}+\left[\frac{N}{2}\right]\right)$, there exist non-vanishing coefficients $\alpha^{i j}$ such that

$$
\bigwedge^{j_{0}(\mathfrak{s o}(N))} \xi_{1}=\bigwedge^{j_{0}(\mathfrak{s o}(N))}\left(a^{i j} d \omega_{i j}\right) \neq 0 .
$$

Taking the 2 -form $\xi_{1}+d \omega_{T}$, the wedge products can be rewritten as a sum

$$
\bigwedge^{p}\left(\xi_{1}+d \omega_{T}\right)=\sum_{k=1}^{p}\left(\begin{array}{l}
p \\
k
\end{array}\right)\left(\bigwedge^{k} \xi_{1}\right) \wedge\left(\bigwedge^{p-k} d \omega_{T}\right) .
$$

The conditions (36) and (37) imply that $k \leq j_{0}(\mathfrak{s o}(N))$ and $p-k \leq 2 N+1$, respectively. This means that $j_{0}(I H a(N)) \leq j_{0}(\mathfrak{s o}(N))+2 N+1$. On the other hand, since $\xi_{1}$ and $d \omega_{T}$ have no terms in common, it follows at once that

$$
\bigwedge^{j_{0}(\mathfrak{s o}(N))+2 N+1} \xi_{1}+d \omega_{T} \neq 0,
$$

showing that

$$
j_{0}(I H a(N))=\frac{N(N-1)}{2}-\frac{1}{2}\left[\frac{N}{2}\right]+2 N+1 .
$$

Applying formula (8) we finally get

$$
\mathcal{N}(\text { IHa }(N))=\left[\frac{N}{2}\right]+1 .
$$

The latter equation shows that adding the new generators $\left\{Q_{k}, P_{k}, E, T\right\}$ has no consequence on the number of invariants.

We conclude that the conditions to apply the generalized ansatz developed earlier are satisfied. Observe that since $T$ is a central element of $I H a(N)$ (and the only invariant of the radical), a power of it must be the function $f$ multiplying the rotation generators $J_{i j}$ in the operators generating the virtual copy. 
Proposition 3 For any $N \geq 3$, the operators

$J_{i j}^{\prime}=J_{i j} T^{2}+\left(G_{i} Q_{j}-G_{j} Q_{i}\right) T+\left(F_{i} P_{j}-F_{j} P_{i}\right) T+\left(P_{i} Q_{j}-P_{j} Q_{i}\right) R$

generate a virtual copy of $\mathfrak{s o}(N)$ in the enveloping algebra of IHa $(N)$. The Casimir operators of the latter are obtained as the symmetrized polynomials of the functions $C_{k}$ arising as coefficients of the characteristic polynomial

$$
\operatorname{Cas}_{N}=\operatorname{det}\left|A_{N}-T \operatorname{Id}_{N}\right|=\sum_{l=1}^{N} C_{2 l} T^{2 N-2 l}+T^{2 N},
$$

where $A_{N}$ is the matrix (30).

The proof is straightforward and follows by direct computation. From (29) and (31) we get that $R, T$ commute with all generators but $E$, which does not appear in the expression of the operators $J_{i j}^{\prime}$. To verify equations (17) and (18), it suffices to compute the commutators of the basis elements $\left\{J_{i j}, G_{k}, F_{k}, Q_{k}, P_{k}, R, E, T\right\}$ with the quadratic polynomials $\left(G_{i} Q_{j}-G_{j} Q_{i}\right),\left(F_{i} P_{j}-F_{j} P_{i}\right)$ and $\left(P_{i} Q_{j}-P_{j} Q_{i}\right)$. We omit the explicit computations, which are mechanical but quite lengthy. The main commutators to reproduce these commutators are given in Appendix A.

\subsection{Invariants of the centrally extended Hamilton algebras}

According to the quantum formulation, the realization of physical states and the projective representations of relativity groups are equivalent to unitary representations of central extensions. Therefore the interesting object to be studied in the context of noninertial states are the central extension of the inhomogeneous Hamilton group [19]. It can be easily proved by means of cohomological tools [20] that the inhomogeneous Hamilton algebra $\operatorname{IHa}(N)$ admits a three dimensional central extension denoted by $Q H a(N)$. The new central generators $L, M, A$ are related to time-energy and position momentum relation, the mass generator and reciprocal symmetry, respectively. $\mathrm{QHa}(\mathrm{N})$ is the maximal centrally extended Lie algebra obtained from $\mathrm{IHa}(\mathrm{N})$. Taking a basis $\left\{J_{i j}, G_{k}, F_{k}, P_{k}, Q_{k}, R, E, T, L, A, M\right\}$, where $L, A, M$ are the generators of the (maximal) central extension, the commutation relations are given by

$$
\begin{aligned}
& {\left[J_{i j}, J_{k l}\right]=\delta_{i}^{l} J_{j k}+\delta_{j}^{k} J_{i l}-\delta_{j}^{l} J_{i k}-\delta_{i}^{k} J_{j l},\left[J_{i j}, G_{k}\right]=-\delta_{i}^{k} G_{j}+\delta_{k}^{j} G_{i},} \\
& {\left[J_{i j}, F_{k}\right]=-\delta_{i}^{k} F_{j}+\delta_{k}^{j} F_{i},\left[J_{i j}, P_{k}\right]=-\delta_{i}^{k} P_{j}+\delta_{k}^{j} P_{i},\left[J_{i j}, Q_{k}\right]=-\delta_{i}^{k} Q_{j}+\delta_{k}^{j} Q_{i},} \\
& {\left[G_{i}, F_{j}\right]=\delta_{i}^{j} R,\left[G_{i}, Q_{j}\right]=\delta_{i}^{j} T,\left[F_{i}, P_{j}\right]=\delta_{i}^{j} T,\left[P_{i}, Q_{j}\right]=\delta_{i}^{j} L,\left[G_{i}, P_{j}\right]=\delta_{i}^{j} M,} \\
& {\left[F_{i}, Q_{j}\right]=\delta_{i}^{j} A,\left[E, G_{i}\right]=-P_{i},\left[E, F_{i}\right]=Q_{i},[E, T]=-L,[E, R]=2 T .}
\end{aligned}
$$

Again, the use of differential forms allows us to compute the number of invariants for arbitrary values of $N$.

Lemma 2 For any $N \geq 3$ the following equality holds

$$
\mathcal{N}(Q H a(N))=\left[\frac{N}{2}\right]+4 \text {. }
$$


Proof. The proof is essentially a consequence of the argument in Lemma 1. For $Q H a(N)$ the Maurer-Cartan equations are

$$
\begin{aligned}
& d \omega_{i j}=-\sum_{k=1}^{N} \omega_{i k} \wedge \omega_{j k}, d \theta_{k}=\sum_{j=1}^{N} \omega_{k j} \wedge \theta_{j}, d \eta_{k}=\sum_{j=1}^{N} \omega_{k j} \wedge \eta_{j}, \\
& d \varphi_{k}=\sum_{j=1}^{N} \omega_{k j} \wedge \varphi_{j}+\omega_{E} \wedge \eta_{k}, d \chi_{k}=\sum_{j=1}^{N} \omega_{k j} \wedge \chi_{j}-\omega_{E} \wedge \theta_{k}, \\
& d \omega_{R}=\sum_{k=1}^{N} \theta_{k} \wedge \eta_{k}, d \omega_{T}=\sum_{k=1}^{N}\left(\theta_{k} \wedge \varphi_{k}+\eta_{k} \wedge \chi_{k}\right)+2 \omega_{E} \wedge \omega_{R}, \\
& d \omega_{L}=\sum_{k=1}^{N} \chi_{k} \wedge \varphi_{k}-\omega_{E} \wedge \omega_{T}, d \omega_{M}=\sum_{k=1}^{N} \theta_{k} \wedge \chi_{k}, \\
& d \omega_{A}=\sum_{k=1}^{N} \eta_{k} \wedge \varphi_{k}, d \omega_{E}=0 .
\end{aligned}
$$

Since $\{L, M, A\}$ are central elements, the forms $\omega_{A}, \omega_{M}$ and $\omega_{L}$ do not appear in the terms of a generic element of $\mathcal{L}(Q H a(N))$. Therefore, the same 2-form used in Lemma 1 provides the maximal rank of an element in $\mathcal{L}(\mathrm{QHa}(\mathrm{N}))$. We thus conclude that

$j_{0}(Q H a(N))=\frac{1}{2}\left(\frac{N(N-1)}{2}-\left[\frac{N}{2}\right]+4 N+2\right)$ and again, by formula (8), we obtain the number of invariants

$\mathcal{N}(Q H a(N))=\frac{N^{2}+7 N+12}{2}-\left(\frac{N(N-1)}{2}-\left[\frac{N}{2}\right]+4 N+2\right)=\left[\frac{N}{2}\right]+4$.

The problem therefore reduces to compute the $\left[\frac{N}{2}\right]+1$ non-central invariants. Observe that, according to Theorem 1, one of these invariants must be an invariant depending only on the generators of the radical. Moreover, in contrast to the invariants of the inhomogeneous Hamilton algebra, which depended upon all its generators, for the quantum Hamilton $\mathrm{QHa}(\mathrm{N})$ algebra we find an important property that turns out to be essential to find closed formulae for the invariants.

Lemma 3 For any generalized Casimir invariant $\Phi$ of $Q H a(N)$ the constraint

$$
\frac{\partial \Phi}{\partial e}=0
$$

is satisfied.

It follows at once from the representation (2) that the differential operator associated to the generator $R$ is given by

$$
\widehat{R}=-2 t \frac{\partial}{\partial e}
$$

thus the invariants do not depend on the variable $e$. An important consequence of this fact is that the generators $\{T, R, M, L, A\}$ commute with all other remaining generators $J_{i j}, G_{k}, F_{k}, Q_{k}, P_{k}$. This will be crucial in finding new operators in the enveloping algebra that generate a virtual copy of $\mathfrak{s o}(N)$.

Proposition 4 For any $N \geq 3$, the operators

$$
\begin{aligned}
\widehat{J}_{i j}=J_{i j}\left(T^{2}+\right. & R L-A M)+T\left(G_{i} Q_{j}-G_{j} Q_{i}\right)+T\left(F_{i} P_{j}-F_{j} P_{i}\right)+L\left(G_{i} F_{j}-G_{j} F_{i}\right)+ \\
& +R\left(P_{i} Q_{j}-P_{j} Q_{i}\right)+M\left(Q_{i} F_{j}-Q_{j} F_{i}\right)+A\left(P_{i} G_{j}-P_{j} G_{i}\right),
\end{aligned}
$$

generate a virtual copy of $\mathfrak{s o}(N)$ in the enveloping algebra of $Q H a(N)$. 
Table 1. Generators of virtual copies of $\mathfrak{s o}(n)$ in the enveloping algebras of one and two dimensional central extensions of $\mathrm{IHa}(\mathrm{N})$.

\begin{tabular}{|c|c|}
\hline $\mathfrak{g}$ & Operators $\widehat{J}_{i j}$ generating the virtual copy of $\mathfrak{s o}(N)$ in $\mathfrak{U}(\mathfrak{g})$ \\
\hline$I H a(n) \uplus\langle L\rangle$ & $\begin{array}{l}J_{i j}\left(T^{2}+R L\right)+T\left(G_{i} Q_{j}-G_{j} Q_{i}+F_{i} P_{j}-F_{j} P_{i}\right)+L\left(G_{i} F_{j}-G_{j} F_{i}\right) \\
+R\left(P_{i} Q_{j}-P_{j} Q_{i}\right)\end{array}$ \\
\hline$I H a(n) \uplus\langle M\rangle$ & $\begin{array}{l}J_{i j} T^{2}+T\left(G_{i} Q_{j}-G_{j} Q_{i}+F_{i} P_{j}-F_{j} P_{i}\right)+M\left(Q_{i} F_{j}-Q_{j} F_{i}\right) \\
+R\left(P_{i} Q_{j}-P_{j} Q_{i}\right)\end{array}$ \\
\hline$I H a(n) \uplus\langle A\rangle$ & $\begin{array}{l}J_{i j} T^{2}+T\left(G_{i} Q_{j}-G_{j} Q_{i}+F_{i} P_{j}-F_{j} P_{i}\right)+A\left(P_{i} G_{j}-P_{j} G_{i}\right) \\
+R\left(P_{i} Q_{j}-P_{j} Q_{i}\right)\end{array}$ \\
\hline$I H a(n) \uplus\langle A, M\rangle$ & $\begin{array}{l}J_{i j}\left(T^{2}-A M\right)+T\left(G_{i} Q_{j}-G_{j} Q_{i}+F_{i} P_{j}-F_{j} P_{i}\right)+R\left(P_{i} Q_{j}-P_{j} Q_{i}\right) \\
+M\left(Q_{i} F_{j}-Q_{j} F_{i}\right)+A\left(P_{i} G_{j}-P_{j} G_{i}\right)\end{array}$ \\
\hline$I H a(n) \uplus\langle A, L\rangle$ & $\begin{array}{l}J_{i j}\left(T^{2}+R L\right)+T\left(G_{i} Q_{j}-G_{j} Q_{i}+F_{i} P_{j}-F_{j} P_{i}\right)+L\left(G_{i} F_{j}-G_{j} F_{i}\right) \\
+R\left(P_{i} Q_{j}-P_{j} Q_{i}\right)+A\left(P_{i} G_{j}-P_{j} G_{i}\right)\end{array}$ \\
\hline$I H a(n) \uplus\langle L, M\rangle$ & $\begin{array}{l}J_{i j}\left(T^{2}+R L\right)+T\left(G_{i} Q_{j}-G_{j} Q_{i}+F_{i} P_{j}-F_{j} P_{i}\right)+L\left(G_{i} F_{j}-G_{j} F_{i}\right) \\
+R\left(P_{i} Q_{j}-P_{j} Q_{i}\right)+M\left(Q_{i} F_{j}-Q_{j} F_{i}\right)\end{array}$ \\
\hline
\end{tabular}

The proof, once more, follows by direct verification of the conditions (17) and (18). As before, we omit the long verification. The main commutators of quadratic polynomials in the generators are given in Appendix A.

We concluide that a fundamental set of invariants of $Q H a(N)$ for arbitrary $N$ is given by $\left\{L, A, M, T^{2}+R N-A M, \operatorname{Sym}\left(C_{2 l}^{\prime}\right)\right\}$, where $\operatorname{Sym}\left(C_{2 l}^{\prime}\right)$ denotes the symmetrization of the polynomials $C_{2 l}$ obtained inserting the operators (45) into the matrix (30) and computing the characteristic polynomial.

We already observed that $Q H a(N)$ is a maximal central extension of $I H a(N)$. The remaining extensions are either one dimensional, with central generator $L, A$ or $M$, or two dimensional, with central charges $\{L, A\},\{L, M\}$ or $\{A, M\}$. In view of the preceding results, it seems reasonable to think that slight modifications of (45) will also provide the corresponding operators that generate the virtual copy of $\mathfrak{s o}(N)$ in the enveloping algebra of these extensions. The Maurer-Cartan equations can be easily obtained from (44), and using them it can be easily shown that $\mathcal{N}(\operatorname{IHa}(N) \uplus\langle\Lambda\rangle)=\left[\frac{N}{2}\right]+2$ and $\mathcal{N}(\operatorname{IHa}(N) \uplus\langle\Lambda, \Pi\rangle)=\left[\frac{N}{2}\right]+3$ for $\Lambda, \Pi \in\{A, M, L\}$. The operators generating the virtual copies are given in Table 1.

\section{Applications to contractions}

Considering that the proposed method is motivated by the problem of finding the invariants of semidirect products, it is natural to ask whether the procedure of virtual copies can be extended to contractions of Lie algebras, in order to obtain a systematic method to compute the invariants of the contraction without being forced to consider the invariants separately [21, 22]. In this section we outline a possible approach to the 
contraction problem. Recall that a contraction of a Lie algebra $\mathfrak{g}$ is determined by a family of non-singular linear maps $\Phi_{\varepsilon}$ of $\mathfrak{g}$, where $\varepsilon \in(0,1]$. If the limit

$$
[X, Y]_{\infty}:=\lim _{\varepsilon \rightarrow 0} \Phi_{\varepsilon}^{-1}\left[\Phi_{\varepsilon}(X), \Phi_{\varepsilon}(Y)\right]
$$

exists for any $X, Y \in \mathfrak{g}$, then equation (46) defines a Lie algebra $\mathfrak{g}^{\prime}$ called the contraction of $\mathfrak{g}\left(\right.$ by $\left.\Phi_{\varepsilon}\right)[23]$.

Suppose that for the semidirect product $\mathfrak{g}=\mathfrak{s} \vec{\oplus}_{R} \mathfrak{r}$ we can find a virtual copy of $\mathfrak{s}$ in the enveloping algebra $\mathcal{U}(\mathfrak{g})$ generated by the operators

$$
X_{i}^{\prime}=X_{i} f\left(Y_{1}, . ., Y_{m}\right)+P_{i}\left(Y_{1}, . ., Y_{m}\right)
$$

satisfying the requirements of (16). Now let $\mathfrak{g} \rightsquigarrow \mathfrak{g}^{\prime}=\mathfrak{s} \vec{\oplus}_{R} \mathfrak{r}^{\prime}$ be a nontrivial generalized Inönü-Wigner contraction such that $\mathcal{N}(\mathfrak{g})=\mathcal{N}\left(\mathfrak{g}^{\prime}\right)$, given by transformations of the type [22]:

$$
\begin{aligned}
& \Phi_{\varepsilon}\left(X_{i}\right)=X_{i}, \\
& \Phi_{\varepsilon}\left(Y_{j}\right)=\varepsilon^{n_{i}} Y_{j}, \quad n_{i} \in \mathbb{Z},
\end{aligned}
$$

where $\left\{X_{1}, . ., X_{n}, Y_{1}, . . Y_{m}\right\}$ is a basis of $\mathfrak{g}$. This means that the contraction is performed only in the radical, preserving the Levi part $\mathfrak{s}$ and the representation $R$. Rewriting the polynomials $f$ and $P_{i}$ over the transformed basis, we obtain the expressions

$$
\begin{aligned}
& f\left(\Phi_{\varepsilon}\left(Y_{1}\right), . ., \Phi_{\varepsilon}\left(Y_{m}\right)\right)=\varepsilon^{-\left(n_{i_{1}}+\ldots+n_{i_{k-1}}\right)} \alpha^{i_{1} \ldots i_{k-1}} Y_{i_{1} \ldots Y_{i_{k-1}},}, \\
& P_{i}\left(\Phi_{\varepsilon}\left(Y_{1}\right), . ., \Phi_{\varepsilon}\left(Y_{m}\right)\right)=\varepsilon^{-\left(n_{j_{1}}+\ldots+n_{j_{k-1}}\right)} \beta_{i}^{j_{1} \ldots j_{k}} Y_{j_{1}} \ldots Y_{j_{k}} .
\end{aligned}
$$

Considering the maximal exponents of the contraction parameter $\varepsilon$ defined by

$$
\begin{aligned}
& M_{0}=\max \left\{n_{i_{1}}+\ldots+n_{i_{k-1}} \mid \alpha^{i_{1} . i_{k-1}} \neq 0\right\}, \\
& M_{i}=\max \left\{n_{i_{1}}+\ldots+n_{i_{k}} \mid \beta_{i}^{i_{1} . . i_{k-1}} \neq 0\right\},
\end{aligned}
$$

we can compute the following limits for $i=1, . ., n$ :

$$
\begin{aligned}
& f_{0}\left(Y_{1}, . ., Y_{m}\right)=\lim _{\varepsilon \rightarrow 0} \varepsilon^{M_{0}} f\left(\Phi_{\varepsilon}\left(Y_{1}\right), . ., \Phi_{\varepsilon}\left(Y_{m}\right)\right)=\sum_{n_{i_{1}}+\ldots+n_{i_{p}}=M_{0}} \alpha^{i_{1} \ldots i_{k-1}} Y_{i_{1}} \ldots Y_{i_{k-1}}, \\
& P_{i, 0}\left(Y_{1}, . ., Y_{m}\right)=\lim _{\varepsilon \rightarrow 0} \varepsilon^{M_{i}} P_{i}\left(\Phi_{\varepsilon}\left(Y_{1}\right), . ., \Phi_{\varepsilon}\left(Y_{m}\right)\right)=\sum_{n_{i_{1}}+\ldots+n_{i_{p}}=M_{i}} \beta_{i}^{j_{1} \ldots j_{k}} Y_{j_{1}} \ldots Y_{j_{k}} .
\end{aligned}
$$

We further introduce the scalars

$$
N_{i}=\max \left\{M_{0}, M_{i}\right\}, \quad i=1, . ., n .
$$

This enables us to compute the following limits for arbitrary index $i$

$$
X_{i}^{\prime \prime}=\lim _{\varepsilon \rightarrow 0} \varepsilon^{N_{i}}\left\{X_{i} f\left(\Phi_{\varepsilon}\left(Y_{1}\right), . ., \Phi_{\varepsilon}\left(Y_{m}\right)\right)+P_{i}\left(\Phi_{\varepsilon}\left(Y_{1}\right), . ., \Phi_{\varepsilon}\left(Y_{m}\right)\right)\right\} .
$$

Observe that if $M_{0}>M_{i_{0}}$ for some $i_{0}$, then the previous limit reduces to

$$
X_{i_{0}}^{\prime \prime}=X_{i} f_{0}\left(Y_{1}, . ., Y_{m}\right) \text {, }
$$

while for $M_{0}<M_{i_{0}}$, equation (51) simplifies to

$$
X_{i_{0}}^{\prime \prime}=P_{i_{0}, 0}\left(Y_{1}, . ., Y_{m}\right) \text {. }
$$

In any case, it is obvious that $f_{0}$ is a Casimir operator of the contraction $\mathfrak{g}^{\prime}$, depending only on the variables of the radical $\mathfrak{r}^{\prime}$. 
Proposition 5 A generalized Inönü-Wigner contraction $\mathfrak{g} \rightsquigarrow \mathfrak{g}^{\prime}$ of type (47) determines a virtual copy of $\mathfrak{s}$ in the enveloping algebra of $\mathfrak{g}^{\prime}$ only if

$$
M_{0}=M_{1}=. .=M_{n} .
$$

The proof is essentially an adaptation of well known facts on contractions of Casimir operators [24]. Suppose that for some index $i_{0} \in\{1, . ., n\}$ we have $N_{i_{0}}=M_{0}$. As commented above, the contracted operator is $X_{i_{0}}^{\prime \prime}=X_{i_{0}} f_{0} \neq 0$. Since the representation $R$ of $\mathfrak{s}$ is preserved by the contraction, there exists at least an element $Y_{k_{0}} \in \mathfrak{r}^{\prime}$ such that $\left[X_{i_{0}}, Y_{k_{0}}\right] \neq 0$. But this implies that

$$
\left[X_{i_{0}}^{\prime \prime}, Y_{k_{0}}\right]=\left[X_{i_{0}} f_{0}, Y_{k_{0}}\right]=\left[X_{i_{0}}, Y_{k_{0}}\right] f_{0} \neq 0
$$

contradicting condition (17). Thus the $\left\{X_{i}^{\prime \prime}\right\}$ cannot generate a virtual copy of $\mathfrak{s}$ in $\mathcal{U}(\mathfrak{g})$. If on the contrary, $N_{i_{0}}=M_{i_{0}}$, then the contraction of the operator $X_{i_{0}}^{\prime}$ equals $X_{i_{0}}^{\prime \prime}=P_{i_{0}, 0}$, which depends only on the variables of $\mathfrak{r}^{\prime}$. Now $\mathfrak{s}$ is semisimple, thus we can find $X_{k_{1}}, X_{k_{2}} \in \mathfrak{s}$ such that $\left[X_{k_{1}}, X_{k_{2}}\right]=X_{i_{0}}$. In this case we would obtain

$$
\left[X_{k_{1}}^{\prime \prime}, X_{k_{2}}^{\prime \prime}\right]=X_{i_{0}} f_{0}^{2}+\ldots \neq X_{i_{0}}^{\prime \prime},
$$

which again prevents the operators $\left\{X_{i}^{\prime \prime}\right\}$ to generate a virtual copy of $\mathfrak{s}$. Observe that this is precisely what happens if the contraction is an inhomogeneous algebra, i.e., $\mathfrak{r}^{\prime}$ is an Abelian algebra.

Let us therefore suppose that all indices $N_{i}$ coincide, i.e., that $M_{0}=M_{i}$ for all $i$. Let $f_{1}=\sum_{n_{i_{1}}+\ldots+n_{i_{p}}<M_{0}} \alpha^{i_{1} \ldots i_{k-1}} Y_{i_{1} \ldots} \ldots Y_{i_{k-1}}$ and $P_{i, 1}=\sum_{n_{i_{1}}+\ldots+n_{i_{p}}<M_{i}} \beta_{i}^{j_{1} \ldots j_{k}} Y_{j_{1} \ldots Y_{j_{k}}}$. For any $i$ we can decompose $\varepsilon^{M_{0}} X_{i}^{\prime}$ with respect to the parameter $\varepsilon$ as

$$
\begin{aligned}
\varepsilon^{M_{0}} X_{i}^{\prime} & =\varepsilon^{M_{0}}\left\{X_{i} f\left(\Phi_{\varepsilon}\left(Y_{1}\right), . ., \Phi_{\varepsilon}\left(Y_{m}\right)\right)+P_{i}\left(\Phi_{\varepsilon}\left(Y_{1}\right), . ., \Phi_{\varepsilon}\left(Y_{m}\right)\right)\right\} \\
& =X_{i}\left(f_{0}+\sum_{p \geq 1} \varepsilon^{M_{0}-p} f_{1}\right)+\left(P_{i, 0}+\sum_{q \geq 1} \varepsilon^{M_{0}-q} P_{i, 1}\right) .
\end{aligned}
$$

Now, over the transformed basis the condition (17) must hold for any $\varepsilon \neq 0$. Developing the commutator $\left[\varepsilon^{M_{0}} X_{i}^{\prime}, Y_{k}\right]$ and reordering the result with respect to $\varepsilon$ leads to to the expression

$$
\begin{aligned}
{\left[\varepsilon^{M_{0}} X_{i}^{\prime}, Y_{k}\right] } & =\left[X_{i}\left(f_{0}+\sum_{p \geq 1} \varepsilon^{M_{0}-p} f_{1}\right)+\left(P_{i, 0}+\sum_{q \geq 1} \varepsilon^{M_{0}-q} P_{i, 1}\right), Y_{k}\right] \\
& =\left[X_{i}^{\prime \prime}, Y_{k}\right]+\sum_{p \geq 1} \varepsilon^{M_{0}-p}\left[X_{i} f_{1}, Y_{k}\right]+\sum_{q \geq 1} \varepsilon^{M_{0}-q}\left[P_{i, 1}, Y_{k}\right]=0 .
\end{aligned}
$$

Therefore, for the limit $\varepsilon \rightarrow 0$ we finally get

$$
\lim _{\varepsilon \rightarrow 0}\left[\varepsilon^{M_{0}} X_{i}^{\prime}, Y_{k}\right]=\left[X_{i}^{\prime \prime}, Y_{k}\right]=0,
$$

which shows that the contracted operators $\left\{X_{i}^{\prime \prime}\right\}$ satisfy constraint (17). Arguing in similar manner, it is shown that constraint (18) is also satisfied. We conclude that the $\left\{X_{i}^{\prime \prime}\right\}$ generate a virtual copy of $\mathfrak{s}$ in the enveloping algebra of the contraction $\mathfrak{g}^{\prime}$. In the particular case of $\mathcal{N}(\mathfrak{g})=\mathcal{N}\left(\mathfrak{g}^{\prime}\right)=\mathcal{N}(\mathfrak{s})+1$, a fundamental set of invariants is completely determined by the corresponding virtual copies and the Casimir operators $f$ and $f_{0}$, respectively. 
As an example to illustrate the contraction of virtual copies, let us consider four boson pairs $\left\{a_{i}, a_{i}^{\dagger}\right\}$ with well-known relations

$$
\left[a_{i}, a_{j}^{\dagger}\right]=\delta_{i j},\left[a_{i}, a_{j}\right]=\left[a_{i}^{\dagger}, a_{j}^{\dagger}\right]=0 .
$$

Let $\mathfrak{g}$ be the Lie algebra generated by the operators

$$
\begin{array}{llll}
X_{1,1}=a_{1}^{\dagger} a_{1}+a_{2}^{\dagger} a_{2}, & X_{-1,1}=a_{1} a_{1}+a_{2} a_{2}, & X_{1,-1}=a_{1}^{\dagger} a_{1}^{\dagger}+a_{2}^{\dagger} a_{2}^{\dagger}, & G_{1}=a_{1} a_{4}+a_{2} a_{3}, \\
F_{1}=a_{2}^{\dagger} a_{3}+a_{1}^{\dagger} a_{4}, & Q_{1}=a_{2} a_{4}+a_{1} a_{3}, & P_{1}=a_{1}^{\dagger} a_{3}+a_{2}^{\dagger} a_{4} & R=a_{4} a_{4}+a_{3} a_{3}, \\
E=a_{3}^{\dagger} a_{4}+a_{4}^{\dagger} a_{3}, & T=2 a_{3} a_{4} .
\end{array}
$$

It is straightforward to verify that the subalgebra generated by $\left\{X_{1,1}, X_{-1,1}, X_{1,-1}, F_{1}, G_{1}, R\right\}$ is isomorphic to the semidirect $\operatorname{sum} \mathfrak{w} \mathfrak{s p}(1, \mathbb{R})$, which incidentally is isomorphic to the 2-photon algebra [18, 25]. We can therefore consider $\mathfrak{g}$ as a kind of "inhomogenization" of $\mathfrak{w} \mathfrak{s} \mathfrak{p}(1, \mathbb{R})$, in analogy with the Hamilton algebras. $\mathfrak{g}$ clearly satisfies $\mathcal{N}(\mathfrak{g})=2$, and although the centre is zero \pm the polynomial $R^{2}-T^{2}$ is a Casimir operator of $\mathfrak{g}$. It can be easily verified that a virtual copy of the Levi subalgebra $\mathfrak{s u}(1,1)$ in the enveloping algebra of $\mathfrak{g}$ is generated by the operators

$$
\begin{aligned}
& X_{1,1}^{\prime}=X_{1,1}\left(R^{2}-T^{2}\right)+T\left(Q_{1} F_{1}+G_{1} P_{1}\right)-R\left(G_{1} F_{1}+Q_{1} P_{1}\right), \\
& X_{-1,1}^{\prime}=X_{-1,1}\left(R^{2}-T^{2}\right)+2 T G_{1} Q_{1}-R G_{1}^{2}-R Q_{1}^{2}, \\
& X_{1,-1}^{\prime}=X_{1,-1}\left(R^{2}-T^{2}\right)+2 T F_{1} P_{1}-R F_{1}^{2}-R P_{1}^{2} .
\end{aligned}
$$

In particular, the second Casimir operator of $\mathfrak{g}$ is easily obtained as

$$
C=\left(X_{1,1}^{\prime}\right)^{2}-\frac{1}{2}\left(X_{-1,1}^{\prime} X_{1,-1}^{\prime}+X_{1,-1}^{\prime} X_{-1,1}^{\prime}\right)
$$

If we now consider the contraction $\mathfrak{g} \rightsquigarrow \mathfrak{g}^{\prime}$ determined by the transformations

$$
\Phi_{\varepsilon}\left(Q_{1}\right)=\varepsilon Q_{1}, \Phi_{\varepsilon}\left(P_{1}\right)=\varepsilon P_{1}, \Phi_{\varepsilon}(E)=\varepsilon E, \Phi_{\varepsilon}(T)=\varepsilon T,
$$

where the remaining generators remain unchanged, we obtain an algebra $\mathfrak{g}^{\prime}$ with two invariants that also extends the 2-photon algebra. Expressing the operators generating the virtual copy in $\mathcal{U}(\mathfrak{g})$ over the transformed basis, and considering the previous limits (51), we obtain

$$
\begin{aligned}
& \varepsilon^{2} X_{1,1}^{\prime}=X_{1,1}\left(\varepsilon^{2} R^{2}-T^{2}\right)+T\left(Q_{1} F_{1}+G_{1} P_{1}\right)-R\left(\varepsilon^{2} G_{1} F_{1}+Q_{1} P_{1}\right), \\
& \varepsilon^{2} X_{-1,1}^{\prime}=X_{-1,1}\left(\varepsilon^{2} R^{2}-T^{2}\right)+2 T G_{1} Q_{1}-R \varepsilon^{2} G_{1}^{2}-R Q_{1}^{2} \\
& \varepsilon^{2} X_{1,-1}^{\prime}=X_{1,-1}\left(\varepsilon^{2} R^{2}-T^{2}\right)+2 T F_{1} P_{1}-R \varepsilon^{2} F_{1}^{2}-R P_{1}^{2} .
\end{aligned}
$$

The contracted operators $X_{i, j}^{\prime \prime}$ are therefore

$$
\begin{aligned}
& X_{1,1}^{\prime \prime}=X_{1,1}\left(-T^{2}\right)+T\left(Q_{1} F_{1}+G_{1} P_{1}\right)-R Q_{1} P_{1}, \\
& X_{-1,1}^{\prime \prime}=X_{-1,1}\left(-T^{2}\right)+2 T G_{1} Q_{1}-R Q_{1}^{2}, \\
& X_{1,-1}^{\prime \prime}=X_{1,-1}\left(-T^{2}\right)+2 T F_{1} P_{1}-R P_{1}^{2} .
\end{aligned}
$$

It is straightforward to verify that these operators generate a copy of $\mathfrak{s u}(1,1)$ in the enveloping algebra of the contraction $\mathfrak{g}^{\prime}$. In particular, the second Casimir operator follows from the expression $C^{\prime}=\left(X_{1,1}^{\prime \prime}\right)^{2}-\frac{1}{2}\left(X_{-1,1}^{\prime \prime} X_{1,-1}^{\prime \prime}+X_{1,-1}^{\prime \prime} X_{-1,1}^{\prime \prime}\right)$, and coincides with the contraction of the Casimir operator $C$ of $\mathfrak{g}$.

\footnotetext{
+ The commutators of $\mathfrak{g}$ and the contraction $\mathfrak{g}^{\prime}$ are given in Appendix B.
} 


\section{Final remarks}

We have seen that modifications of the procedure to compute the Casimir operators of semidirect products $\mathfrak{s} \vec{\oplus}_{R} \mathfrak{w}(n)$ using quadratic operators in the enveloping algebra can be extended naturally to more general types of Lie algebras, including semidirect products with pure solvable radicals. This enables us to derive the Casimir operators of such Lie algebras using the classical formulae for the invariants of semisimple algebras. The conditions (17) and (18) also determine the range of validity of the ansatz based on operators of the form (16). First of all, the semidirect product $\mathfrak{g}=\mathfrak{s} \vec{\oplus}_{R} \mathfrak{r}$ must possess at least $\mathcal{N}(\mathfrak{s})+1$ Casimir operators, one of them depending only on the generators of $\mathfrak{r}$ and $\mathcal{N}(\mathfrak{s})$ depending on all generators of $\mathfrak{g}$. Further, the radical $\mathfrak{r}$ of $\mathfrak{g}$ cannot be Abelian. This implies that the procedure is not directly adaptable to classical inhomogeneous algebras, at least with the proposed type of operators. In spite of this apparent restrictions, the class of Lie algebras to which the method of virtual copies can be applied is ample. It covers the central extensions of semidirect products of the type $\mathfrak{s} \vec{\oplus}_{R} \mathfrak{w}(n)$ (whenever they exist), as well as other "inhomogenizations" containing the latter as subalgebras. In particular, it comprises the one dimensional non-central extensions of double inhomogeneous Lie algebras [26].

We have also shown that for generalized Inönü-Wigner contractions $\mathfrak{g} \rightsquigarrow \mathfrak{g}^{\prime}=\mathfrak{s} \vec{\oplus}_{R} \mathfrak{r}^{\prime}$ that preserve the Levi subalgebra $\mathfrak{s}$ and the representation $R$, the procedure can be applied to construct a virtual copy of $\mathfrak{s}$ in the enveloping algebra of $\mathfrak{g}^{\prime}$. In some sense, this result can be interpreted as a kind of contraction of realizations of semisimple Lie algebras. In particular, from this we easily deduce the Casimir operators of the contraction in compact form. This leads to the question whether the ansatz can be reversed, i.e., under which conditions the realization of a simple Lie algebra $\mathfrak{s}$ in the enveloping algebra of a semidirect product $\mathfrak{g}^{\prime}$ can be deformed into a realization in another enveloping algebra $\mathcal{U}(\mathfrak{g})$, and whether the underlying Lie algebras $\mathfrak{g}$ and $\mathfrak{g}^{\prime}$ are related by contraction [24. This problem is deeply related with the stability problem of semidirect products of Lie algebras, a question that remains largely unsolved.

\section{Acknowledgment}

During the preparation of this work, the first author (RCS) was financially supported by the research project MTM2006-09152 of the M.E.C. and the project and CCG07UCM/ESP-2922 of the U.C.M.-C.A.M.

\section{References}

[1] de Shalit A and Talmi I 1963 Nuclear Shell Theory (Academic Press, N. Y.) Frank A and van Isacker P 1994 Algebraic Models in Molecular and Nuclear Structure Physics (J. Wiley, N.Y.) Iachello F 2006 Lie Algebras and Applications (Springer Verlag, N.Y.)

[2] Seiberg N and Witten E 1994 Nucl. Phys. B426 19 
Dmitrasinovic V 2001 Phys. Lett. B 499135

[3] Racah G 1950 Rend. Sci. Fis. Mat. Nat. 8108

[4] Gel'fand I M 1950 Mat. Sb. 50103

Gruber B and O'Raifeartaigh L 1964 J. Math. Phys. 51796

Perelomov A M and Popov V S 1968 Izv. Akad. Nauk 321368

de Azcárraga J A, Perelomov A M and Pérez Bueno J C 1996 J. Phys. A: Math. Gen. 297993

Karaday H R and Gungormez M 1997 J. Math. Phys. 386976

de Azcárraga J A, Macfarlane A J, Mountain A J and Pérez Bueno J C 1998 Nucl. Phys. B510 657

[5] Macfarlane A J and Pfeiffer H 2001 J. Math. Phys. 413192

de Azcárraga J A and Macfarlane A J 2001 J. Math. Phys. 42419

[6] Cornwell J F 1984 Group Theory in Physics (Academic Press, N. Y.)

Georgi H 1999 Lie Algebras in Particle Physics (Perseus Books, Reading, MA)

[7] Beltrametti E G and Blasi A 1966 Phys. Lett. 2062

Mutze U 1969 Z. Physik 229224

[8] Abellanas L and Martínez Alonso L 1975 J. Math. Phys. 161580

[9] Peccia A and Sharp R T 1976 J. Math. Phys. 171313

[10] Campoamor-Stursberg R 2003 J. Phys. A: Math. Gen. 361357

[11] Campoamor-Stursberg R 2003 Phys. Lett. A312 211

[12] Boyko V, Patera J and Popovych R 2006 J. Phys. A: Math. Gen. 395749

2007 J. Phys. A: Math. Theor. 40113

[13] Campoamor-Stursberg R 2008 J. Phys. A: Math. Gen. 41365207

[14] Low S G 2007 J. Phys. A: Math. Gen. 403999

[15] Low S G 2008 J. Phys. A: Math. Gen. 41304034

[16] Campoamor-Stursberg R 2007 J. Phys. A: Math. Theor. 4014773

[17] Campoamor-Stursberg R 2004 Phys. Lett. A327 138

[18] Quesne Ch 1988 J. Phys. A: Math. Gen. 21 L321

[19] Low S G 2007 J. Math. Phys. 48102901

[20] de Azcárraga J A, Izquierdo J M 1995 Lie Groups, Lie Algebras, Cohomology and some Applications to Physics (Cambridge: Cambridge Univ. Press)

[21] Vitiello G and de Concini C 1976 Nuclear Phys. B116 141

Celeghini E, Tarlini M and Vitiello G 1984 Nuovo Cimento A 8419

[22] Weimar-Woods E 2000 Rev. Math. Phys. 121505

[23] Inönü E and Wigner E P 1953 Proc. Natl. Acad. Sci. U.S. 39510

Saletan E 1961 J. Math. Phys. 21

Kupczyński M 1969 Comm. Math. Phys. 13154

[24] Frank A, Alhassid Y and Iachello F 1986 Phys. Rev. A34 677

Herranz F J and Santander M 1997 J. Phys. A: Math. Gen. 305411

Campoamor-Stursberg R 2005 J. Phys. A: Math. Gen. 384187

[25] Zhang W M, Feng D H and Gilmore R 1990 Rev. Mod. Phys. 62867

Ballesteros A, Herranz F J and Parashar 1997 J. Phys. A: Math. Gen. 308587

Ballesteros A and Herranz F J 2007 J. Phys. A: Math. Theor. 40 F51

[26] Herranz F J, Pérez Bueno J C and Santander M 1998315327

Campoamor-Stursberg R 2006 J. Phys. A: Math. Gen. 392325 


\section{Appendix A}

In this appendix we specify the commutators of the basis elements of $\mathfrak{g}$ with higher order polynomials in the generators. The relations are presented simultaneously for the inhomogeneous Hamilton algebra $\mathrm{IHa}(\mathrm{N})$ and its various central extensions. To obtain the corresponding commutators for e.g. I $\mathrm{Ha}(\mathrm{N})$, all terms containing the generators $M, A, L$ in the following list are skipped. Analogous procedure holds for the remaining extensions.

$$
\begin{aligned}
& {\left[J_{i j}, G_{k} Q_{l}\right]=\delta_{j}^{k} G_{i} Q_{l}-\delta_{i}^{k} G_{j} Q_{l}+\delta_{j}^{l} G_{k} Q_{i}-\delta_{l}^{i} G_{k} Q_{j},} \\
& {\left[J_{i j}, F_{k} P_{l}\right]=\delta_{j}^{k} F_{i} P_{l}-\delta_{i}^{k} F_{j} P_{l}+\delta_{j}^{l} F_{k} P_{i}-\delta_{l}^{i} F_{k} P_{j} \text {, }} \\
& {\left[J_{i j}, G_{k} F_{l}\right]=\delta_{j}^{k} G_{i} F_{l}-\delta_{i}^{k} G_{j} F_{l}+\delta_{j}^{l} G_{k} F_{i}-\delta_{l}^{i} G_{k} F_{j},} \\
& {\left[J_{i j}, P_{k} Q_{l}\right]=\delta_{j}^{k} P_{i} Q_{l}-\delta_{i}^{k} P_{j} Q_{l}+\delta_{j}^{l} P_{k} Q_{i}-\delta_{l}^{i} P_{k} Q_{j},} \\
& {\left[J_{i j}, Q_{k} F_{l}\right]=\delta_{j}^{k} Q_{i} F_{l}-\delta_{i}^{k} Q_{j} F_{l}+\delta_{j}^{l} Q_{k} F_{i}-\delta_{l}^{i} Q_{k} F_{j},} \\
& {\left[J_{i j}, P_{k} G_{l}\right]=\delta_{j}^{k} P_{i} G_{l}-\delta_{i}^{k} P_{j} G_{l}+\delta_{j}^{l} P_{k} G_{i}-\delta_{l}^{i} P_{k} G_{j},} \\
& {\left[G_{i} Q_{j}, G_{k} Q_{l}\right]=\delta_{i}^{l} T G_{k} Q_{j}-\delta_{k}^{j} T G_{i} Q_{l} \text {, }} \\
& {\left[G_{i} Q_{j}, F_{k} P_{l}\right]=\delta_{i}^{k} R Q_{j} P_{l}-\delta_{l}^{j} L G_{i} F_{k}+\delta_{i}^{l} M F_{k} Q_{j}+\delta_{k}^{i} \delta_{j}^{l} R L,} \\
& {\left[G_{i} Q_{j}, G_{k} F_{l}\right]=\delta_{i}^{l} R G_{k} Q_{j}-\delta_{j}^{k} T G_{i} F_{l}-\delta_{l}^{j} A G_{i} G_{k} \text {, }} \\
& {\left[G_{i} Q_{j}, P_{k} Q_{l}\right]=\delta_{i}^{l} T P_{k} Q_{j}-\delta_{j}^{k} L G_{i} Q_{l}+\delta_{i}^{k} M Q_{j} Q_{l},} \\
& {\left[G_{i} Q_{j}, Q_{k} F_{l}\right]=\delta_{i}^{k} T Q_{j} F_{l}-\delta_{j}^{l} A G_{i} Q_{k}+\delta_{i}^{k} \delta_{j}^{l} T A \text {, }} \\
& {\left[G_{i} Q_{j}, P_{k} G_{l}\right]=\delta_{i}^{k} M Q_{j} G_{l}-\delta_{j}^{k} L G_{i} G_{l}-\delta_{l}^{j} T G_{i} P_{k}+\delta_{k}^{i} \delta_{l}^{j} M T \text {, }} \\
& {\left[F_{i} P_{j}, F_{k} P_{l}\right]=\delta_{i}^{l} T F_{k} P_{j}-\delta_{k}^{j} T F_{i} P_{l},} \\
& {\left[F_{i} P_{j}, G_{k} F_{l}\right]=-\delta_{j}^{l} T F_{i} G_{j}-\delta_{j}^{k} M F_{i} F_{l}-\delta_{k}^{j} R P_{j} F_{k}-\delta_{k}^{i} \delta_{l}^{j} R T,} \\
& {\left[F_{i} P_{j}, P_{k} Q_{l}\right]=\delta_{j}^{l} L F_{i} P_{k}+\delta_{i}^{k} T Q_{l} P_{j}+\delta_{i}^{l} A P_{j} P_{k},} \\
& {\left[F_{i} P_{j}, Q_{k} F_{l}\right]=\delta_{k}^{j} L F_{i} F_{l}-\delta_{l}^{j} T F_{i} Q_{k}+\delta_{k}^{i} A F_{l} P_{j},} \\
& {\left[F_{i} P_{j}, Q_{k} F_{l}\right]=\delta_{k}^{j} L F_{i} F_{l}-\delta_{l}^{j} T F_{i} Q_{k}+\delta_{i}^{k} A F_{l} P_{j},} \\
& {\left[F_{i} P_{j}, P_{k} G_{l}\right]=\delta_{k}^{i} T P_{j} G_{l}-\delta_{l}^{j} M P_{k} F_{i}-\delta_{l}^{i} R P_{k} P_{j},} \\
& {\left[G_{i} F_{j}, G_{k} F_{l}\right]=\delta_{l}^{i} R G_{k} F_{j}-\delta_{j}^{k} R G_{i} F_{l} \text {, }} \\
& {\left[G_{i} F_{j}, P_{k} Q_{l}\right]=\delta_{k}^{j} T G_{i} Q_{l}+\delta_{j}^{l} A G_{i} P_{k}+\delta_{k}^{i} M Q_{l} F_{j}+\delta_{i}^{l} T P_{k} F_{j},} \\
& {\left[G_{i} F_{j}, Q_{k} F_{l}\right]=\delta_{k}^{j} A G_{i} F_{l}+\delta_{k}^{i} T F_{j} F_{l}+\delta_{i}^{l} R Q_{k} F_{j}} \\
& {\left[G_{i} F_{j}, P_{k} G_{l}\right]=\delta_{k}^{j} T G_{i} G_{l}-\delta_{l}^{j} R G_{i} P_{k}+\delta_{k}^{i} M F_{j} G_{l}+\delta_{k}^{i} \delta_{j}^{l} M R,} \\
& {\left[P_{i} Q_{j}, P_{k} Q_{l}\right]=\delta_{l}^{i} L P_{k} Q_{j}-\delta_{k}^{j} L P_{i} Q_{l} \text {, }} \\
& {\left[P_{i} Q_{j}, Q_{k} F_{l}\right]=\delta_{k}^{i} L F_{l} Q_{j}-\delta_{l}^{j} A P_{i} Q_{k}-\delta_{l}^{i} T Q_{k} Q_{j},} \\
& {\left[P_{i} Q_{j}, P_{k} G_{l}\right]=-\delta_{k}^{j} L P_{i} G_{l}-\delta_{l}^{j} T P_{i} P_{k}-\delta_{i}^{l} M P_{k} Q_{j},} \\
& {\left[Q_{i} F_{j}, Q_{k} F_{l}\right]=\delta_{k}^{j} A Q_{i} F_{l}-\delta_{l}^{i} A Q_{k} F_{v},} \\
& {\left[Q_{i} F_{j}, P_{k} Q_{l}\right]=\delta_{k}^{j} T Q_{i} G_{l}-\delta_{i}^{k} L F_{j} G_{l}-\delta_{l}^{j} R P_{k} Q_{i}-\delta_{i}^{l} T P_{k} F_{j},} \\
& {\left[P_{i} G_{j}, P_{k} G_{l}\right]=\delta_{k}^{j} M P_{i} G_{l}-\delta_{i}^{l} M P_{k} G_{j} \text {, }} \\
& {\left[P_{i} G_{j}, E\right]=P_{i} P_{j}, \quad\left[T^{2}+R L, E\right]=0,} \\
& {\left[T G_{i} Q_{j}, E\right]=T P_{i} Q_{j}+L G_{i} Q_{j}, \quad\left[R P_{i} Q_{j}, E\right]=-2 T P_{i} Q_{j} .}
\end{aligned}
$$




\section{Appendix B}

The Lie algebra $\mathfrak{g}$ spanned by the operators $\left\{X_{1,1}, X_{-1,1}, X_{1,-1}, F_{1}, G_{1}, Q_{1}, P_{1}, R, E, T\right\}$ has Levi decomposition

$$
\mathfrak{g}=\mathfrak{s u}(1,1) \vec{\oplus}_{R} \mathfrak{r},
$$

where $R=D_{\frac{1}{2}} \oplus D_{\frac{1}{2}} \oplus D_{0}^{3}, D_{\frac{1}{2}}$ being the two dimensional irreducible spin $\frac{1}{2}$-representation and $D_{0}$ the trivial representation. The commutators of $\mathfrak{g}$ and the contraction $\mathfrak{g}^{\prime}$ are simultaneously given in the following table, for the values of $\alpha=1$ and $\alpha=0$, respectively.

\begin{tabular}{c|cccccccccc}
{$[]$,} & $X_{1,1}$ & $X_{-1,1}$ & $X_{1,-1}$ & $G_{1}$ & $F_{1}$ & $Q_{1}$ & $P_{1}$ & $R$ & $E$ & $T$ \\
\hline$X_{1,1}$ & 0 & $-2 X_{-1,1}$ & $2 X_{1,-1}$ & $-G_{1}$ & $F_{1}$ & $-Q_{1}$ & $P_{1}$ & 0 & 0 & 0 \\
$X_{-1,1}$ & & 0 & $4 X_{1,1}$ & 0 & $2 G_{1}$ & 0 & $2 Q_{1}$ & 0 & 0 & 0 \\
$X_{1,-1}$ & & & 0 & $-2 F_{1}$ & 0 & $-2 P_{1}$ & 0 & 0 & 0 & 0 \\
$G_{1}$ & & & & 0 & $R$ & 0 & $T$ & 0 & $Q_{1}$ & 0 \\
$F_{1}$ & & & & & 0 & $-T$ & 0 & 0 & $P_{1}$ & 0 \\
$Q_{1}$ & & & & & & 0 & $\alpha R$ & 0 & $\alpha G_{1}$ & 0 \\
$P_{1}$ & & & & & & & 0 & 0 & $\alpha F_{1}$ & 0 \\
$R$ & & & & & & & & 0 & $2 T$ & 0 \\
$E$ & & & & & & & & & 0 & $-2 \alpha R$ \\
$T$ & & & & & & & & & & 0
\end{tabular}

